Original Article

Artigo Original

\begin{abstract}
Alessandra Spada Durante ${ }^{1}$ Margarita Bernal Wieselberg ${ }^{1}$

Sheila Carvalho

Nayara Costa ${ }^{1}$

Beatriz Pucci ${ }^{1}$

Nicolly Gudayol ${ }^{1}$

Kátia de Almeida ${ }^{1}$

Keywords

Evoked Potentials, Auditory Cortical Potential Speech Detection

Hearing Aids

Electrophysiology
\end{abstract}

Descritores

Potenciais Evocados Auditivos

Percepção de Fala

Percepção Auditiva

Auxiliares de Audição

Eletrofisiologia

Correspondence address:

Alessandra Spada Durante

Rua Dr. Cesário Mota Júnior, 61, $8^{\circ}$ andar, São Paulo (SP), Brasil,

CEP: 01221-020.

E-mail: alessandra.durante@fcmsantacasasp.edu.br

Received: 19/03/2014

\section{Cortical Auditory Evoked Potential: evaluation of speech detection in adult hearing aid users}

\author{
Potencial Evocado Auditivo Cortical: avaliação da \\ detecção de fala em adultos usuários de prótese auditiva
}

\begin{abstract}
Purpose: To analyze the presence of auditory cortical potential and its correlation with psychoacoustic detection of speech sounds as well as the latency of the P1, N1 e P2 components presented in free field in hearing impaired adults with and without amplification. Methods: We evaluated 22 adults with moderate to severe symmetrical bilateral sensorineural hearing loss, regular users of bilateral hearing aids. Speech sounds of low $(/ \mathrm{m} /)$, medium $(/ \mathrm{g} /)$ and high $(/ \mathrm{t} /)$ frequencies were presented in sound field in decreasing intensities of 75, 65 and of $55 \mathrm{dBSPL}$ in free field with and without hearing aids. The used equipment performs automatic statistical detection of the presence of response; forthermore, the latencies of waves P1, N1 e P2 were labeled and the psychoacoustic perception was registered. Results: The results demonstrated the increased presence of cortical response with hearing aids. We observed the correlation between psychoacoustic perception and automatic detection of $91 \%$ for the sounds $/ \mathrm{g} /$ and $/ \mathrm{t} /$ and ranged from 73 to $86 \%$ for the sound $/ \mathrm{m} /$. The averages of latencies P1-P2-N1 decreased with both increasing intensity and the use of hearing aids for the three sounds. The differences were significant for the sounds /g/ and /t/ in comparison with and without hearing aids. Conclusion: There was increase in the presence of cortical auditory evoked potential with hearing aids. Automatic detection of cortical response provided with hearing aids showed $91 \%$ agreement with the psychoacoustic perception of the speech signal. In the analysis of latency measures of the P1, N1 and P2 components, it was observed a decrease with the increase of the signal intensity and the use of amplification for the three speech stimuli /m/, /g/ and /t/.
\end{abstract}

\section{RESUMO}

Objetivo: Analisar a presença do potencial auditivo cortical e sua concordância com a detecção psicoacústica de sons de fala bem como a latência dos componentes P1, N1 e P2 apresentados em campo livre em adultos deficientes auditivos com e sem o uso da amplificação. Métodos: Foram avaliados 22 adultos com perda auditiva sensorioneural bilateral simétrica de grau moderado a severo, usuários regulares de prótese auditiva bilateral. Foram utilizados estímulos de fala de baixas (/m/), médias (/g/) e altas (/t/) frequências, apresentados em intensidades decrescentes de 75, 65 e $55 \mathrm{dBNPS}$ em campo livre nas condições sem e com o uso de próteses auditivas. O equipamento utilizado realiza a detecção estatística automática da resposta; além disso, as latências das ondas P1, N1 e P2 foram marcadas e a percepção psicoacústica foi registrada. Resultados: Os resultados demonstraram o aumento da presença de resposta cortical na condição com prótese auditiva. Observou-se a concordância entre a percepção psicoacústica e a detecção automática de $91 \%$ para os sons /g/ e /t/ e variou de 73 a $86 \%$ para o som /m/. As médias das latências P1, N1 e P2 diminuíram tanto com o aumento da intensidade como com o uso da prótese auditiva para os três sons de fala. As diferenças foram significantes para os sons /g/ e/t/ na comparação sem e com prótese auditiva. Conclusão: Houve aumento da presença do potencial evocado auditivo cortical na condição com prótese auditiva. A detecção automática da presença de resposta cortical na condição com prótese auditiva apresentou $91 \%$ de concordância com a percepção psicoacústica do sinal de fala. Na análise das medidas de latência dos componentes P1, N1 e P2, observou-se a uma diminuição tanto com o aumento da intensidade do sinal como com o uso da amplificação para os três estímulos de fala /m/, /g/e /t/.

Study carried out at the School of Medical Sciences, Santa Casa de São Paulo - FCMSCSP - São Paulo (SP), Brazil. (1) Speech Language Pathology and Audiology Course, School of Medical Sciences, Santa Casa de São Paulo FCMSCSP - São Paulo (SP), Brazil.

Source of funding: São Paulo Research Foundation (FAPESP).

Conflict of interests: nothing to declare. 


\section{INTRODUCTION}

The auditory evoked potentials (AEPs) are electrical modifications in the auditory nervous system, generated in several levels of the central auditory nervous system by an acoustic stimulus. They are classified according to latency (interval of time between the presentation of the sound stimulus and wave peak) into three groups: short-latency potentials, occurring in the first $10-12 \mathrm{~ms}$; middle-latency potentials, occurring between 12 and $50 \mathrm{~ms}$; and long-latency potentials, also called cortical potentials, occurring between 50 and $600 \mathrm{~ms}^{(1)}$.

The most common exogenous cortical auditory evoked potentials (CAEPs) are P1, N1 e P2. These components provide information concerning the arrival of sound information to the auditory cortex ${ }^{(2)}$. For an adult, the P1 wave is characterized by a small positive peak, with latency of approximately $50 \mathrm{~ms}$ after stimulus; one high negative peak (N1), about $100 \mathrm{~ms}$ after the stimulus begins; and one second high positive peak (P2), about $200 \mathrm{~ms}$ after stimulus. The auditory assessment using the CAEPs presents several advantages, because it allows evaluating the whole auditory system (from the brain stem to the cortex) and because it can be registered among awake participants, obtained with a variety of acoustic stimuli presented both with headphones and in free field, which is an especially favorable situation ${ }^{(1,3)}$. Even if the clinical and scientific value of these potentials is inestimable, the daily use of these cortical potentials were compromised in the past, due to several factors. The pattern of response of the main components of CAEP substantially changes depending on the level of development from birth to adolescence. It is also possible to observe great variability concerning range, latency, and morphology of inter- and intrasubject responses; there is also variance concerning the type and the characteristics of the stimulus. Therefore, by considering the significant maturational changes occurring in CAEP throughout development, the major variability of responses, besides the technical limitation of electrodes, filters, and amplifiers to capture these potentials, added to the difficulty to interpret responses and the high costs of the equipment, the clinical use of CAEP has been hampered until recently ${ }^{(3,4)}$.

The increasing number of infants, who were diagnosed early by neonatal auditory screening programs, being referred to the adaptation of hearing aids created challenges related to speech language pathology and audiology. The first one involves ensuring a reliable estimation of hearing thresholds of this population. The second one involves the calculation of electro-acoustic characteristics and the necessary adjustments, based on these hearing thresholds, using prescriptive formulas, ensuring that speech signals can be detected in an optimized, effective, and comfortable manner for this child ${ }^{(5)}$. Whether the prescribed targets of acoustic gain and maximum outputs are being reached can be observed with adequate prescriptive formulas, punctually and individually, to measure the performance of a specific hearing-aid device. However, these formulas depend on the accuracy of calculations based on the measured or estimated hearing threshold. Errors, deviations, or imprecision that may occur for any of these stages can compromise the goals of amplification to be reached. The larger the deviation, the harder it is to ensure the expected benefits or that the provided sounds would, in fact, reach the auditory cortex ${ }^{(6,7)}$. Besides, with the objective of adapting performance and comfort of amplification, reports given by the patient are used to make fine adjustments of electroacoustic features of amplification. However, in case of newborns or adults who cannot respond, who are going through the process of adaptation of hearing aids, besides the observation of clinical behavior and statements from parties in charge, we do not have any objective validation means that can ensure this patient can detect the amplified sound, especially the speech sound in normal conversation intensities. With this impasse, it was possible to observe increasing interest for the CAEP, which was revitalized by studies that showed its clinical use in various scenarios of auditory, learning, mental, and neurological changes ${ }^{(8)}$.

Recent studies ${ }^{(3,9-12)}$ show that the CAEPs obtained from speech stimuli are promising for the hearing rehabilitation of individuals, because they provide information referring to the objective record of speech stimuli detection and processing in the auditory cortex, with or without a hearing-aid device. Therefore, the assessment of cortical responses would lead to important complementary information about the perception (or the lack of perception) of the amplified sound.

On the basis of these findings, the National Acoustic Laboratories (NAL), a governmental institution from Australia, has developed, in the past few years, a device to study cortical potentials, named HEARLab ${ }^{\circledR}$. This system is accessible for clinical use, because of its advanced technology and ability to reduce noise and interferences is also more sensitive to capture responses and counts on an exclusive method of statistical detection and automatic analysis of responses, therefore, it does not require a subjective interpretation from the examiner ${ }^{(3,10,13,14)}$, thus it is different from similar devices. Besides, the equipment allows the manual peak marking and analysis of aspects related to latency and amplitude of responses, which are equally present in traditional research devices of the CAEPs.

The hypothesis of this study is that it is possible to safely measure the effects of sound amplification for speech stimuli in cortical measures by means of the automatic analysis equipment of the CAEPs. In Brazil, there are no studies made with devices that provide the automatic analysis of CAEPs. Therefore, the objective of this analysis was to verify the presence of cortical auditory potential and its agreement with the psychoacoustic detection of speech sounds, as well as the latency of components P1, N1 and P2 presented in a free field for adults with hearing impairment, with or without hearing aids.

\section{METHODS}

The research project was approved by the Ethics Research Committee of the School of Medical Sciences, in Santa Casa de São Paulo (FCMSCSCP), protocol \#. 327/10. All the participants were informed about the objectives of the study and the informed consent was obtained. 
In this study, 22 adults (aged between 19 and 76 years) with bilateral hearing loss were assessed; 16 were female and 6 were male participants, selected from the database of the Speech Language Pathology and Audiology clinic of Santa Casa de São Paulo.

Adult or elderly individuals, with moderate to severe sensorineural, bilateral, symmetric hearing loss, wearing bilateral hearing aid for at least 1 year were included in the study. Individuals with mixed, conductive, or unilateral hearing loss, or presenting with other neurological or psychiatric conditions were not included in the study.

The descriptive analysis of the sample as to age, level of loss, time of use, and aspects of the hearing aid is presented in Chart 1.

To develop the study, we used an audiometer (model Itera, GN Otometrics) and a middle ear analyzer (model AZ 7R, Interacoustics). Audiometry tests were classified as mild, moderate, moderately severe, severe, and profound, according to the criterion by Davis and Silverman ${ }^{(15)}$.

The HEARLab ${ }^{\circledR}$ system was used to detect the CAEPs. The system has one model used to assess if speech signals produce a cortical response among hearing-aid users (Aided Cortical Assessment). In this module, the device enables three different speech stimuli: $/ \mathrm{m} /, / \mathrm{g} /$, and $/ \mathrm{t} /$, which were selected for allowing spectral emphasis in low, medium, and high frequencies, respectively ${ }^{(3,10,13)}$. These phonemes were extracted from the continuous speech of a female speaker. They were filtered to coincide with the International LongTerm Average Speech Spectrum (ILTASS). A high-pass filter was also applied with $250 \mathrm{~Hz}$ in the sounds of /t/ and /g/ to remove unwanted low-frequency noises. The three sounds provide information about speech perception in different regions of frequencies. The device has three testing intensities: 55, 65, and $75 \mathrm{dBNPS}$, which represent signals of weak, medium, and strong speech intensity, respectively, presented in free field by a sound box.

The equipment has a system of automatic analysis (multivariate analysis of varianceMANOVA), which objectively determines if there is a cortical response or not. The significance level (p-level) generated by the system indicates the presence of response when p-value is lower than 0.05 .

At first, the protocol adopted to characterize the participant was filled out, containing data concerning general personal information, hearing health and aspects related to the use of a hearing aid or not. Once inclusion and exclusion criteria were analyzed, the evaluation protocol was initiated.

Chart 1. Characteristics of the sample as to age, sex, level of loss and hearing-aid device

\begin{tabular}{|c|c|c|c|c|c|c|}
\hline \multirow{2}{*}{ Subject } & \multirow{2}{*}{ Age } & \multirow{2}{*}{ Sex } & \multirow{2}{*}{ Level of loss } & \multicolumn{3}{|c|}{ Hearing-aid device } \\
\hline & & & & Type & Model & Daily use (hours) \\
\hline 1 & 48 & $\mathrm{~F}$ & Moderate & Retro & HIT & 12 \\
\hline 2 & 49 & $\mathrm{~F}$ & Moderate/severe & Retro & NEO 112 & 12 \\
\hline 3 & 40 & $\mathrm{~F}$ & Moderate & Retro & LNQ65D & 12 \\
\hline 4 & 49 & $\mathrm{~F}$ & Moderate/severe & Intra & Element 8 & 8 \\
\hline 5 & 36 & $\mathrm{~F}$ & Moderate & Retro & Dual M7 & 12 \\
\hline 6 & 59 & M & Moderate/severe & Retro & Hit & 8 \\
\hline 7 & 42 & $\mathrm{~F}$ & Moderate/severe & Retro & ZG71D & 12 \\
\hline 8 & 19 & $\mathrm{~F}$ & Moderate & Retro & UNA SP AZ & 14 \\
\hline 9 & 57 & $\mathrm{~F}$ & Moderate & Retro & A74 & 12 \\
\hline 10 & 53 & M & Severe & Retro & One35 & 8 \\
\hline 11 & 39 & $\mathrm{~F}$ & Moderate/severe & Retro & LNQ65D & 8 \\
\hline 12 & 59 & $\mathrm{~F}$ & Moderate/severe & Retro & Hit & 10 \\
\hline 13 & 26 & $\mathrm{~F}$ & Moderate/severe & Intra & Extra 11 & 8 \\
\hline 14 & 63 & M & Moderate/severe & Retro & LNQ75D & 10 \\
\hline 15 & 26 & $\mathrm{~F}$ & Severe & Retro & IDT35 & 14 \\
\hline 16 & 54 & $\mathrm{~F}$ & Moderate & Retro & Essential & 8 \\
\hline 17 & 25 & $\mathrm{~F}$ & Moderate & Intra & Bravíssimo & 10 \\
\hline 18 & 55 & M & Moderate/severe & Retro & NEO 112 & 12 \\
\hline 19 & 49 & M & Moderate & Retro & Dual M7 & 14 \\
\hline 20 & 56 & $\mathrm{~F}$ & Moderate/severe & Intra & $\mathrm{B} 2 \mathrm{X}$ & 12 \\
\hline 21 & 51 & M & Moderate & Retro & ZG61D & 12 \\
\hline 22 & 76 & $\mathrm{~F}$ & Moderate & Retro & $770 \mathrm{D}$ & 8 \\
\hline
\end{tabular}

Caption: Retro $=$ retroauricular; Intra $=$ intrauricular; $\mathrm{F}=$ female; $\mathrm{M}=$ male 
All the participants went through the same evaluation protocol, which included the performance of pure tone air and bone audiometry, acoustic immittance measures, verification of the adequate functioning of hearing aids, and record of CAEPs under aided and unaided condition in free field.

Before cortical response evaluation, behavioral detection was registered for each one of the speech stimuli under the same testing conditions used to assess the CAEP.

After placing the electrodes: vertex (CZ), right or left mastoids (M1 and M2), and the ground electrode on the forehead (FZ), participants were assessed while being alert, placed at an azimuth of $0^{\circ}$ with relation to the sound box. They were distracted by images on a soundless television, in an acclimatized room that was acoustically treated.

The three speech stimuli of low $(/ \mathrm{m} /)$, middle $(/ \mathrm{g} /)$, and high $(/ t /)$ frequencies were presented in decreasing intensities of 75 , 65 , and $55 \mathrm{dBNPS}$, beginning without the use of hearing aids. The same sequence was adopted with hearing aids, which were previously checked as to their functioning. Their programming for current use, with all the algorithms for signal processing, was activated.

The latency of waves P1, N1, and P2 were also manually marked in the responses considered by the equipment as being present $(\mathrm{p}<0.05)$, both with and without hearing aids. Marking was in accordance with the visual orientation provided by the HEARLab ${ }^{\circledast}$ equipment, being confirmed by an evaluator judge.

Statistical analysis used Mann-Whitney and Wilcoxon tests, as well as the repeated measures analysis of variance (ANOVA). In all the tests, a 0.05 significance level was adopted (or 5\%) to reject the null hypothesis.

\section{RESULTS}

The automatic analysis of the presence of cortical response can be visualized in Table 1. It is possible to observe the increasing presence of response when the hearing aid is being used. In all of the presentations of speech sounds $/ \mathrm{m} /, / \mathrm{g} /$, and $/ \mathrm{t} /$, in the condition of wearing a hearing-aid device, the individual reported sound perception. Therefore, there was agreement between the psychoacoustic perception and the automatic detection, according to the proportion of automatic detection at the presence of response, $91 \%$ for $/ \mathrm{g} /$ and $/ \mathrm{t} /$ speech sounds, and ranging from 73 to $86 \%$ for the $/ \mathrm{m} /$ sound.

Latencies of components P1, N1 and P2 in the different speech sounds and intensities are presented in Tables 2 to 4 (without hearing aid) and in Tables 5 to 7 (with hearing aid). The results showed that the average of latencies of components $\mathrm{P} 1, \mathrm{~N} 1$ and $\mathrm{P} 2$ reduced with the increasing intensity and with the use of hearing-aid devices for the three sounds $/ \mathrm{m} /, / \mathrm{t} /$, and $/ g /$. Differences were significant for sounds $/ \mathrm{t} / \mathrm{and} / \mathrm{g} /$ in the comparison between under aided and unaided conditions, presenting significant tendency for the phoneme $/ \mathrm{m} /$ in the amplified response $(\mathrm{p}>0,06)$.
Figure 1 shows that for the intensity of 75 dBNPS, the latency of components $\mathrm{P} 1, \mathrm{~N} 1$ and $\mathrm{P} 2$ declined with the increasing frequency rate of speech sound, that is, the highest speech stimuli /t/, presented with the lowest latencies, under aided and unaided conditions $(\mathrm{p}<0.01)$.

Table 1. Presence of cortical auditory evoked potential elicited by the phonemes $/ \mathrm{m} /, / \mathrm{g} /$ and $/ \mathrm{t} /$ in intensity levels 55,65 and $75 \mathrm{dBNPS}$, with and without hearing aid devices by the automatic analysis

\begin{tabular}{lccc}
\hline Sound & $\begin{array}{c}\text { Intensity } \\
\text { (dBNPS) }\end{array}$ & $\begin{array}{c}\text { Presence without } \\
\text { hearing aid device (\%) }\end{array}$ & $\begin{array}{c}\text { Presence with hearing } \\
\text { aid device (\%) }\end{array}$ \\
\hline \multirow{3}{*}{$\mathrm{m}$} & 55 & 50.0 & 72.7 \\
& 65 & 50.0 & 86.4 \\
& 75 & 77.3 & 90.9 \\
$\mathrm{~g}$ & 55 & 36.4 & 90.9 \\
& 65 & 54.5 & 90.9 \\
$\mathrm{t}$ & 75 & 90.9 & 90.9 \\
$\mathrm{t}$ & 55 & 63.6 & 90.9 \\
& 65 & 86.4 & 90.9 \\
& 75 & 81.8 & 90.9 \\
\hline
\end{tabular}

Table 2. Descriptive statistics of components $P 1, N 1$, and $P 2$ with the speech stimulus $/ \mathrm{m} /$, under aided and unaided conditions

\begin{tabular}{|c|c|c|c|c|c|}
\hline & \multirow{2}{*}{ Wave } & \multirow{2}{*}{$\begin{array}{l}\text { Intensity } \\
\text { (dBNPS) }\end{array}$} & \multicolumn{3}{|c|}{ Latency (ms } \\
\hline & & & Mean (SD) & Median & Min - Max \\
\hline \multirow{9}{*}{$/ \mathrm{m} /$} & \multirow{3}{*}{$\mathrm{P} 1$} & 55 & $77.0(15.0)$ & 78.5 & $53-102$ \\
\hline & & 65 & $65.2(16.8)$ & 68.0 & $39-86$ \\
\hline & & 75 & $58.2(15.7)$ & 58.0 & $31-94$ \\
\hline & \multirow{3}{*}{$\mathrm{N} 1$} & 55 & $114.3(25.0)$ & 115.5 & $84-168$ \\
\hline & & 65 & $106.9(22.3)$ & 111.0 & $63-139$ \\
\hline & & 75 & $102.9(20.2)$ & 106.0 & $61-145$ \\
\hline & \multirow{3}{*}{ P2 } & 55 & $192.2(19.6)$ & 197.0 & $147-211$ \\
\hline & & 65 & $183.0(11.5)$ & 186.0 & $166-200$ \\
\hline & & 75 & $179.4(24.9)$ & 173.0 & $124-271$ \\
\hline
\end{tabular}

Caption: SD = standard deviation; Min = minimum; $\operatorname{Max}=$ maximum

Table 3. Descriptive statistics of components P1, N1, and P2 with the speech stimulus /t/, under aided and unaided conditions

\begin{tabular}{|c|c|c|c|c|c|}
\hline & \multirow{2}{*}{ Wave } & \multirow{2}{*}{$\begin{array}{l}\text { Intensity } \\
\text { (dBNPS) }\end{array}$} & \multicolumn{3}{|c|}{ Latency (ms } \\
\hline & & & Mean (SD) & Median & Min - Max \\
\hline & & 55 & $66.8(22.7)$ & 57.0 & $32-105$ \\
\hline & P1 & 65 & $60.3(19.7)$ & 55.0 & $31-90$ \\
\hline & & 75 & $47.4(15.1)$ & 47.0 & $26-76$ \\
\hline & & 55 & $108.0(21.1)$ & 109.5 & $69-140$ \\
\hline \multirow[t]{5}{*}{$/ \mathrm{t} /$} & N1 & 65 & $99.0(20.2)$ & 97.0 & $67-161$ \\
\hline & & 75 & $86.4(15.0)$ & 85.0 & $63-110$ \\
\hline & & 55 & $185.3(20.9)$ & 186.0 & $143-222$ \\
\hline & P2 & 65 & $175.4(23.5)$ & 180.0 & $130-214$ \\
\hline & & 75 & $159.3(23.0)$ & 166.0 & $112-190$ \\
\hline
\end{tabular}

Caption: SD = standard deviation; Min = minimum; $\mathrm{Max}=$ maximum 
Table 4. Descriptive statistics of components $\mathrm{P} 1, \mathrm{~N} 1$, and $\mathrm{P} 2$ with speech stimulus $/ \mathrm{g} /$, under aided and unaided conditions

\begin{tabular}{ccccc}
\hline \multirow{2}{*}{ Wave } & Intensity & \multicolumn{3}{c}{ Latency (ms } \\
\cline { 3 - 5 } & (dBNPS) & Mean (SD) & Median & Min - Max \\
\hline \multirow{3}{*}{ P1 } & 55 & $80.3(39.2)$ & 69.0 & $30-151$ \\
& 65 & $55.0(25.7)$ & 53.0 & $27-127$ \\
& 75 & $52.7(20.7)$ & 50.5 & $28-93$ \\
\hline \multirow{3}{*}{ N1 } & 55 & $123.5(42.0)$ & 119.5 & $81-210$ \\
& 65 & $92.2(25.2)$ & 90.5 & $66-155$ \\
& 75 & $88.5(22.2)$ & 86.5 & $53-133$ \\
\hline \multirow{3}{*}{ N1 } & 55 & $186.7(25.0)$ & 185.0 & $147-235$ \\
& 65 & $166.0(20.0)$ & 168.0 & $136-202$ \\
& 75 & $162.9(22.4)$ & 164.5 & $126-211$ \\
\hline
\end{tabular}

Caption: SD = standard deviation; Min = minimum; Max = maximum

Table 5. Descriptive statistics of components P1, N1, and P2 with speech stimulus $/ \mathrm{m} /$, under aided and unaided conditions

\begin{tabular}{|c|c|c|c|c|c|}
\hline & \multirow{2}{*}{ Wave } & \multirow{2}{*}{$\begin{array}{l}\text { Intensity } \\
\text { (dBNPS) }\end{array}$} & \multicolumn{3}{|c|}{ Latency (ms } \\
\hline & & & Mean (SD) & Median & Min - Max \\
\hline \multirow{9}{*}{$/ \mathrm{m} /$} & \multirow{3}{*}{$\mathrm{P} 1$} & 55 & $75.0(19.0)$ & 77.0 & $43-105$ \\
\hline & & 65 & $62.0(14.7)$ & 64.0 & $35-85$ \\
\hline & & 75 & $52.0(14.0)$ & 53.5 & $29-82$ \\
\hline & \multirow{3}{*}{ N1 } & 55 & $118.0(17.3)$ & 119.0 & $87-157$ \\
\hline & & 65 & $109.5(20.4)$ & 109.0 & $73-153$ \\
\hline & & 75 & $93.0(19.7)$ & 96.5 & $35-122$ \\
\hline & \multirow{3}{*}{$\mathrm{P} 2$} & 55 & $191.1(15.9)$ & 189.0 & $167-225$ \\
\hline & & 65 & $184.3(19.1)$ & 184.0 & $140-229$ \\
\hline & & 75 & 177.5 (39.0) & 171.0 & $49-186$ \\
\hline
\end{tabular}

Caption: $\mathrm{SD}=$ standard deviation; Min = minimum; Max = maximum
Table 6. Descriptive statistics of components $\mathrm{P} 1, \mathrm{~N} 1$, and P2 with speech stimulus / $/ \mathrm{t}$, under aided and unaided conditions

\begin{tabular}{ccccc}
\hline \multirow{2}{*}{ Wave } & Intensity & \multicolumn{3}{c}{ Latency (ms } \\
\cline { 3 - 5 } (dBNPS) & Mean (SD) & Median & Min - Max \\
\hline \multirow{3}{*}{ P1 } & 55 & $55.8(17.3)$ & 51.0 & $24-86$ \\
& 65 & $49.7(16.0)$ & 43.5 & $26-85$ \\
& 75 & $41.6(15.0)$ & 38.0 & $18-82$ \\
\hline \multirow{3}{*}{$/ \mathrm{t} / \mathrm{N} 1$} & 55 & $96.9(21.0)$ & 95.5 & $65-135$ \\
& 65 & $87.4(15.8)$ & 90.0 & $60-111$ \\
& 75 & $79.5(16.7)$ & 82.5 & $35-105$ \\
\hline \multirow{3}{*}{ P2 } & 55 & $176.7(24.2)$ & 175.5 & $123-227$ \\
& 65 & $163.0(21.5)$ & 171.0 & $118-191$ \\
& 75 & $152.0(29.9)$ & 158.0 & $50-189$ \\
\hline
\end{tabular}

Caption: $\mathrm{SD}=$ standard deviation; $\mathrm{Min}=$ minimum; $\mathrm{Max}=$ maximum

Table 7. Descriptive statistics of components $\mathrm{P} 1, \mathrm{~N} 1$, and $\mathrm{P} 2$ with speech stimulus /g/, under aided and unaided conditions

\begin{tabular}{|c|c|c|c|c|c|}
\hline & \multirow{2}{*}{ Wave } & \multirow{2}{*}{$\begin{array}{l}\text { Intensity } \\
\text { (dBNPS) }\end{array}$} & \multicolumn{3}{|c|}{ Latency (ms } \\
\hline & & & Mean (SD) & Median & Min - Max \\
\hline \multirow{9}{*}{$/ g /$} & \multirow{3}{*}{ P1 } & 55 & $56.0(16.5)$ & 52.0 & $30-94$ \\
\hline & & 65 & $49.0(14.0)$ & 50.0 & $27-74$ \\
\hline & & 75 & $43.4(13.0)$ & 46.0 & $22-67$ \\
\hline & \multirow{3}{*}{ N1 } & 55 & $92.3(13.2)$ & 90.5 & $70-123$ \\
\hline & & 65 & 87.8 (12.3) & 86.0 & $70-107$ \\
\hline & & 75 & $79.5(10.0)$ & 78.5 & $58-103$ \\
\hline & \multirow{3}{*}{ P2 } & 55 & $171.9(20.3)$ & 174.0 & $130-201$ \\
\hline & & 65 & $166.2(22.2)$ & 162.0 & $128-206$ \\
\hline & & 75 & $155.9(22.0)$ & 156.0 & $115-196$ \\
\hline
\end{tabular}

Caption: $\mathrm{SD}=$ standard deviation; $\mathrm{Min}=$ minimum; $\mathrm{Max}=$ maximum
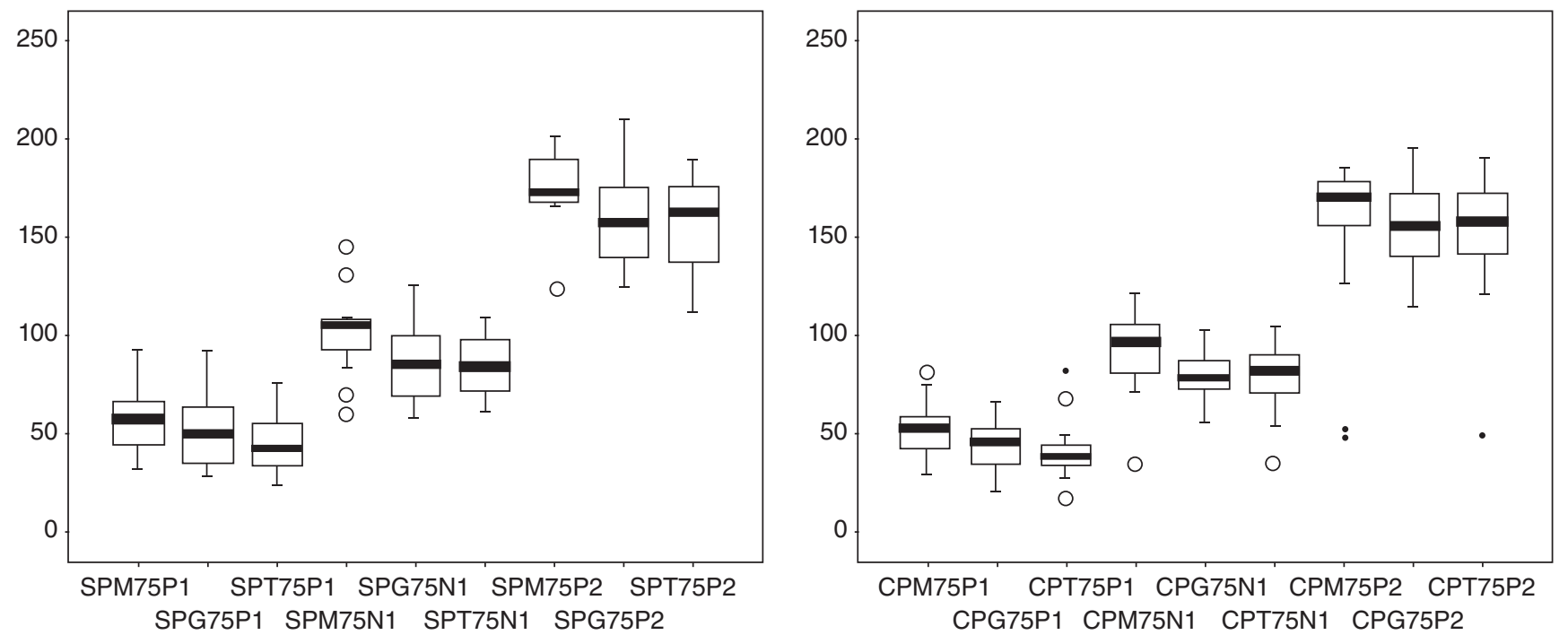

Figure 1. Latency of components P1, N1 e P2 per sound (/m/, /g/, and /t/) with intensity of $75 \mathrm{dBNPS}$, with and without hearing-aid device 


\section{DISCUSSION}

The applications of CAEP in audiological diagnosis have many advantages, especially due to the proximity of its generators with sound perception. This favors the use of complex speech sounds. The acoustic characteristics of complex sounds can be reflected in the form and latency of these potentials; therefore, they can be used to determine the integrity of the neural codification of these characteristics, thus contributing with the determination of speech perception. Recent studies suggest that consonants and vowels have different representations in the central auditory system, both in laboratory animals $^{(16)}$ and in human beings ${ }^{(17)}$.

In this study, results with the use of speech sounds suggest that CAEPs can be used as an objective tool to assess the characteristics of amplification and the validation of hearing-aid fittings, thus enabling the measurement of audibility of speech sound amplification among individuals who cannot collaborate, which is also stated by other authors ${ }^{(3,9,10,18-21)}$, who showed that the use of hearing aids improved the presence of measurable cortical responses (Table 1).

According to Purdy et al. ${ }^{(7)}$, if the hearing-aid device enables speech sounds to generate cortical responses, then the presence of cortical responses provide evidence that the speech sounds received via amplification were detected. Besides, the record of CAEP latencies can be used to follow-up children with hearing impairment using hearing-aid devices or cochlear implants in a clinical environment, thus showing the hearing development through changes in the latency of these potentials ${ }^{(8,21)}$.

\section{Effect of Intensity versus Amplification}

In literature, the effect of intensity is well demonstrated, that is, when the intensity of stimulus increases, the peak of latencies of the components becomes shorter, which means reduced time of neural conduction; and the amplitude increases, which represents increasing strength of response ${ }^{(22)}$.

The reduced latency of components P1, N1 and P2, with the use of hearing-aid devices, is also expected. Such an effect occurs independently if sound is presented in the free field, with or without hearing-aid devices. However, the effect of sound amplification is not so clear. Such a fact could be justified because sound amplification does not have the same effect as the real increase in sound intensity. The hearing-aid device changes the characteristics of the stimulus, as well as the relationship signal/noise, which may affect the capture of AEPs ${ }^{(11,23,24)}$. These findings reinforce the importance of controlling the characteristics of the hearing-aid device and the levels of intensity of the stimulus when the individual is assessed wearing a hearing-aid device. By testing the same person in different evaluation sessions, or even by comparing the response of individuals, it is important to mention different gain adjustments of the hearing device.

Our results showed that the average cortical response in the aided condition was significantly lower for the phoneme $/ \mathrm{m} /$ than for the sounds $/ \mathrm{g} /$ and $/ \mathrm{t} /$. The obtained findings corroborate with those in the literature ${ }^{(19)}$, which also showed responses that were significantly more present in CAEP for the sounds /g/ and $/ \mathrm{t} /$ under aided condition. For the sound $/ \mathrm{m} /$, no significant increase in the amplified response was observed, with fewer responses generated in the amplified condition. One possible explanation may be related to the lower amplification prescribed for low frequencies low frequencies ${ }^{(5,25)}$.

Besides, the analysis of latency values for the intensity of 75 dBNPS seems to show an increasing latency for deeper sounds, with the presence or use of a hearing-aid device (Figure 1). The tonotopic organization is expressed in every auditory pathway ${ }^{(26)}$, and it could explain this effect, which is not described in literature and can be investigated in new studies with the amplification of speech sound use in electrophysiological tests. The use of cortical potentials is adequate for the assessment of amplification due to several reasons, especially because of the possibility of using speech sounds; the longer duration of the spectrum of these speech sounds, which enable the amplified stimulus to have similar performance in relation to its functioning in real life; because it represents sound detection throughout the auditory path, which can be affected by all the parts in the auditory system, as well as the response rate of gain and frequency of the hearing-aid device ${ }^{(7,10)}$.

The chances of using an objective method to analyze the presence or the absence of CAEP will increment the clinical use of this potential. Our results showed that the use of automatic cortical responses made available by the device revealed an agreement between psychoacoustic perception of the sound and the register of the amplified cortical response in $91 \%$ of the presentations, except for the $/ \mathrm{m} /$ sound $(73-86 \%$ ). Recent studies conducted with the application of this type of analysis and device in a population of adults and children ${ }^{(3,13)}$ showed that the results obtained with the automatized analysis of responses were at least equivalent, if not higher, to the analysis of responses that was conducted by an experience human technician.

Therefore, the use of CAEP in hearing aids fitting protocol of adaptation of hearing-aid devices, with the objective of validating amplification, is justified for characterizing an objective measurement that the amplified speech stimulus is able to trigger the neural response in the auditory cortex, therefore, it can be noticed $^{(7)}$. The results in this study confirm the use of CAEPs as a valuable clinical tool to verify hearing-aid fitting in participants that are difficult to be tested, such as children, adults, or elders who are unable to respond. Besides, for audiology control, the CAEPs can be repeated at different times to document different latencies ${ }^{(27,28)}$.

\section{CONCLUSION}

There was an increasing presence of the CAEP among participants using hearing-aid devices; the automatic detection of the presence of cortical response at the presence of hearing-aid devices was in agreement with the psychoacoustic perception of the speech signal in $91 \%$ of presentations for sounds of middle and high frequencies. It was possible 
to observe the effect of intensity under aided and unaided conditions, with the decreasing latencies of components P1, $\mathrm{N} 1$, and $\mathrm{P} 2$, both with the increasing intensity of the signal and in the use of amplification for the three speech stimuli, $/ \mathrm{m} /, / \mathrm{g} /$, and $/ \mathrm{t} /$.

* $S C, N C, B P$, and $N G$ were in charge of data collection and tabulation; $A S D, M B W$, and $K A$ collaborated and supervised data collection and tabulation, as well as data analysis; ASD was responsible for the study project and design, and the general orientation of the steps of execution and elaboration of the manuscript.

\section{REFERENCES}

1. Hall JW. New handbook of auditory evoked responses. Boston: Pearson, Allyn and Bacon; 2007.

2. Näätänen R, Picton T. The N1 wave of the human electric and magnetic response to sound: a review and an analysis of the component structure. Psychophysiology. 1987;24(4):375-425.

3. Golding M, Dillon H, Seymour J, Carter L. The detection of adult cortical auditory evoked potentials (CAEPs) using an automated statistic and visual detection. Int J Audiol. 2009;48(12):833-42.

4. Campbell K, Colrain I. Event-related potential measures of the inhibition of information processing: II. The sleep onset period. Int J Psychophysiol. 2002;46(3):197-214.

5. Ching T, Dillon H. Prescribing amplification for children: adultequivalent hearing loss, real-ear aided gain, and NAL-NL1. Trends Amplif. 2003;7(1):1-9.

6. Dillon H. So, baby, how does it sound? Cortical assessment of infants with hearing aids. Hear J. 2005;58(10):10-7.

7. Purdy SC, Katsch R, Dillon H, Storey L, Sharma M. Aided cortical auditory evoked potentials for hearing instrument evaluation in infants. In: Seewald RC, Bamford JM, editors. A Sound Foundation through Early Amplification. Proceedings of the Third International Conference; 2005. Stafa, Switzerland: Phonak AG; 2005. p. 115-27.

8. Sharma A, Dorman MF, Spahr AJ. A sensitive period for the development of the central auditory system in children with cochlear implants: implications for age of implantation. Ear Hear. 2002;23(6):532-9.

9. Carter L, Dillon H, Seymour J, Seeto M, Van Dun B. Cortical auditoryevoked potentials (CAEPs) in adults in response to filtered speech stimuli. J Am Acad Audiol. 2013;24(9):807-22.

10. Van Dun B, Carter L, Dillon H. Sensitivity of cortical auditory evoked potential (CAEP) detection for hearing-impaired infants in response to short speech sounds. Audiol Res. 2012;2(13):65-76.

11. Billings CJ, Tremblay KL, Souza PE, Binns MA. Effects of hearing aid amplification and stimulus intensity on cortical auditory evoked potentials. Audiol Neurootol. 2007;12(4):234-46.

12. Souza P, Tremblay K. New perspectives on assessing amplification effects. Trends Amplif. 2006;10(3):119-43.
13. Carter L, Golding M, Dillon H, Seymour J. The detection of infant cortical auditory evoked potentials (CAEPs) using statistical and visual detection techniques. J Am Acad Audiol. 2010;21(5):347-56.

14. Munro KJ, Purdy SC, Ahmed S, Begum R, Dillon H. Obligatory cortical auditory evoked potential waveform detection and differentiation using a commercially available clinical system: HEARLab ${ }^{\mathrm{TM}}$. Ear Hear. 2011;32(6):782-6.

15. Davis H, Silverman SR. Auditory Test Hearing Aids. In: Davis H, Silvermann SR, editors. Hearing and deafness. Holt: Rinehart and Winston Hearing and Deafness; 1970.

16. Perez CA, Engineer CT, Jakkamsetti V, Carraway RS, Perry MS, Kilgard MP. Different timescales for the neural coding of consonant and vowel sounds. Cereb Cortex. 2013;23(3):670-83.

17. Kuuluvainen S, Nevalainen P, Sorokin A, Mittag M, Partanen E, Putkinen $\mathrm{V}$, et al. The neural basis of sublexical speech and corresponding nonspeech processing: a combined EEG-MEG study. Brain Lang. 2014;130:19-32.

18. Golding M, Dillon H, Seymour J, Purdy SC, Katsch R. Obligatory Cortical Auditory Evoked Potentials (CAEPs) in infants - a five year review. National Acoustic Laboratories Research \& Development Annual Report 2005/2006. Chatswood, New South Wales, Australia: Australian Hearing; 2006.

19. Chang HW, Dillon H, Carter L, van Dun B, Young ST. The relationship between cortical auditory evoked potential (CAEP) detection and estimated audibility in infants with sensorineural hearing loss. Int $\mathrm{J}$ Audiol. 2012;51(9):663-70.

20. Korczak PA, Kurtzberg D, Stapells DR. Effects of sensorineural hearing loss and personal hearing aids on cortical event-related potential and behavioral measures of speech-sound processing. Ear Hear. 2005;26(2):165-85.

21. Sharma A, Martin K, Roland P, Bauer P, Sweeney MH, Gilley P, et al. P1 latency as a biomarker for central auditory development in children with hearing impairment. J Am Acad Audiol. 2005;16(8):564-73.

22. Adler G, Adler J. Influence of stimulus intensity on AEP components in the 80-to 200 - millisecond latency range. Audiology. 1989;28(6):316-24.

23. Billings CJ, Tremblay KL, Miller CW. Aided cortical auditory evoked potentials in response to changes in hearing aid gain. Int J Audiol. 2011;50(7):459-67.

24. Tremblay KL, Kalstein L, Billings CJ, Souza PE. The neural representation of consonant-vowel transitions in adults who wear hearing aids. Trends Amplif. 2006;10(3):155-62.

25. Dillon H. Hearing aids. Sydney: Boomerang Press; 2001.

26. Van Dijk P, Langers DR. Mapping tonotopy in human auditory cortex. Adv Exp Med Biol. 2013;787:419-25.

27. Alvarenga KF, Vicente LC, Lopes RCF, Silva RA, Banhara MR, Lopes $\mathrm{AC}$, et al. Influência dos contrastes de fala nos potenciais evocados auditivos corticais. Braz J Otorhinolaryngol. 2013;79(3):336-41.

28. Campbell JD, Cardon G, Sharma A. Clinical Application of the P1 Cortical Auditory Evoked Potential Biomarker in Children with Sensorineural Hearing Loss and Auditory Neuropathy Spectrum Disorder. Semin Hear. 2011;32(2):147-55. 